\title{
La dépollution des rejets urbains par temps de pluie : une approche globale
}

\author{
François Guerber*, Jean-Pierre Tabuchi** \\ Agence de l'eau Seine Normandie, 51, rue Salvador Allende, 92027 Nanterre Cedex (1) 47764424 \\ * Directeur des études et recherches \\ ** Responsable du groupe pluvial
}

\section{Introduction}

Bien que reconnue depuis déjà une vingtaine d'années, la pollution par les rejets urbains de temps de pluie ne constituait pas l'un des thèmes d'actions prioritaires des agences. En effet, leurs moyens financiers en matière de dépollution des collectivités locales étaient principalement mobilisés par la lutte contre la pollution par temps sec. S'il reste encore à faire dans ce domaine, des résultats ont été obtenus. C'est d'ailleurs en partie l'amélioration de la qualité du milieu naturel, due à la disparition de certaines pollutions chroniques, qui a permis de mettre en évidence les effets négatifs des rejets urbains par temps de pluie. En particulier on observe des mortalités piscicoles parfois spectaculaires liée à l'amélioration de la qualité de certains cours d'eau qui, de ce fait, s'enrichissent d'une faune et d'une flore plus variées et plus sensibles à des pollutions épisodiques. Aujourd'hui, la reconquête du milieu naturel passe par un maintien de l'effort dans la lutte contre la pollution par temps sec, mais aussi par une prise en considération de la pollution causée par les rejets urbains de temps de pluie.

Ces constatations ont confirmé la nécessité d'une action des agences de l'eau en vue d'obtenir une meilleure maîtrise de la qualité des eaux lors des épisodes pluvieux. Cela s'est traduit par la prise en compte de la dépollution des rejets urbains par temps de pluie dans le $6^{\mathrm{e}}$ programme d'interventions des agences de l'eau. Prise en compte qui se traduira par un soutien financier aux études préalables et aux travaux de dépollution. La politique de l'agence de l'eau Seine-Normandie dans ce domaine s'appuiera donc sur un volet technique et sur un volet financier.

Du point de vue technique, la lutte contre la pollution par les rejets urbains par temps de pluie sera conçue en intégrant différents domaines tels que la construction et l'entretien d'ouvrages de transport et de dépollution, la limitation de la pollution à la source, mais aussi l'urbanisation tant existante que future.

Du point de vue financier, la politique de l'agence sera voisine de celle mise en œuvre au cours du précédent programme concernant l'épuration des collectivités.

\section{Quelques définitions}

Le terme "pluvial» recouvre un grand nombre de significations entraînant une certaine confusion qu'il convient de clarifier par quelques définitions.

Rejets urbains de temps de pluie (RUP) : ce terme recouvre l'ensemble des rejets au milieu naturel occasionnés par les précipitations météoriques en milieu urbain.

Surverses unitaires : il s'agit des rejets occasionnés par les réseaux unitaires lorsque leur capacité de transport est dépassée à la suite d'un épisode pluvieux.

Rejets d'eaux pluviales: rejets occasionnés par les réseaux d'eaux pluviales strictes.

Rejets pseudo-pluviaux : il s'agit de rejets occasionnés par des réseaux d'eaux pluviales dans lesquels transitent des eaux usées provenant d'erreurs de branchements ou de déversoirs d'orages situés plus en amont.

\section{Le volet technique}

Dans le domaine technique, la politique de l'agence s'articule essentiellement autour de quatre points qui sont :

* des études pour orienter les travaux,

* des objectifs de qualité,

* la mise en œuvre d'une politique curative,

* la mise en œuvre d'une politique préventive.

Ces quatre points doivent faire l'objet d'une approche globale. En effet, plus que pour les rejets chroniques de temps sec, les rejets urbains par temps de pluie sont le résultat du cycle de l'eau en milieu urbain dans son ensemble, y compris les eaux usées. Par conséquent une plus large connaissance du bassin versant est nécessaire. Cette connaissance doit intégrer le milieu naturel, la climatologie, les caractéristiques physiques du bassin versant, les données d'urbanisme, etc... 


\subsection{Les études}

Avant d'entreprendre des travaux de lutte contre la pollution par les rejets urbains par temps de pluie, un effort d'études doit être entrepris afin de définir un état des lieux le plus précis possible. En effet, une action efficace doit prendre en compte d'une part tous les facteurs ayant une influence sur la pollution (occupation du sol, activités industrielles, durée de temps sec...) mais également les paramètres influencés par la pollution tels que l'impact sur le milieu naturel ou les conséquences sur les usages de l'eau.

Un inventaire des données disponibles sera fait, complété par des mesures portant d'une part sur le milieu naturel mais également sur les réseaux et la station d'épuration. Elles s'attacheront à préciser la qualité du milieu naturel par temps sec et par temps de pluie en cherchant à déterminer la cause principale de dégradation de la qualité du cours d'eau (MES, DBO5, ammonium, etc...) et l'origine de cette pollution. De même, sur les réseaux et la station il s'agit de préciser le fonctionnement de ces ouvrages par temps de pluie aussi bien que par temps sec. De plus ces études diagnostic devront intégrer de nouveaux paramètres permettant de caractériser la traitabilité des rejets urbains par temps de pluie en procédant, par exemple, à des mesures de décantabilité et à des études granulométriques. Le complément utile à ces mesures est la modélisation mathématique qui sera validée avec les résultats des mesures. Le principal intérêt de ces outils mathématiques est qu'ils permettent de simuler différents scénarios d'évolution de l'urbanisme, de modifications des caractéristiques du réseau etc...

La réflexion doit prendre appui sur les connaissances sur l'impact des rejets urbains par temps de pluie sur le milieu naturel. Pour cela on dispose aujourd'hui d'un certain nombre de connaissances qui permettent de donner une première orientation.

\subsection{Les impacts des rejets urbains par temps de pluie}

\subsubsection{Impact sur les usages de l'eau}

Les usages les plus perturbés par les rejets urbains de temps de pluie sont la fabrication d'eau potable, la pêche et les loisirs.

La fabrication d'eau potable : la fabrication d'eau potable peut être sérieusement perturbée par la présence d'ammonium, de matière en suspension, de produits phytosanitaires et d'hydrocarbures. La gestion des installations de traitement est d'autant plus délicate que l'écart entre la qualité de la ressource en eau par temps de pluie et par temps sec est important.

La pêche: outre les mortalités piscicoles évoquées plus haut, la conchyliculture, la pisciculture et la pêche peuvent être perturbées par les rejets urbains de temps de pluie. En effet, ceux-ci, par leurs apports, entraînent une contamination des différentes espèces de poissons et de mollusques qui peuvent présenter des concentrations en toxiques ou une contamination bactérienne les rendant impropres à la consommation. Par ailleurs la reproduction des différentes espèces peut être altérée.

Le tourisme et les loisirs: les effets des rejets urbains de temps de pluie sur le tourisme ne sont pas négligeables, notamment sur le littoral où les surverses unitaires s'accompagnent d'une pollution microbienne incompatible avec la baignade et les activités conchylicoles. Ces pollutions peuvent se traduire par des interdictions de baignade ou de pêche à pied altérant ainsi l'image de la station balnéaire, ce qui peut avoir des incidences économiques non négligeables.

\subsubsection{Impact sur les écosystèmes}

Les effets des rejets urbains de temps de pluie sur le milieu naturel sont principalement de deux natures: les effets immédiats et les effets différés. Cette séparation en deux classes résulte de la nature des polluants véhiculés par ces rejets : pollution organique carbonée et azotée biodégradable qui créent une baisse rapide de l'oxygénation des eaux, micro-polluants minéraux ou organiques d'une très grande stabilité qui ont la faculté de se concentrer en différents points de l'écosystème.

\subsubsection{Les effets immédiats}

Le premier effet est une augmentation de la turbidité causée par l'apport de matières en suspensions. S'il s'agit uniquement d'apports terrigènes, les effets sont limités: envasement du lit de la rivière, diminution de la transparence de l'eau. Si ces matières en suspensions comportent une forte charge en matière organique, l'impact sur le milieu naturel sera beaucoup plus fort: cet apport de matières organiques induit une consommation d'oxygène qui peut être très importante liée à la biodégradation de la matière organique contenue dans l'effluent, à la décomposition de la matière organique déposée au fond du cours d'eau et à l'oxydation des formes réduites de l'azote. Il s'en suit un déficit en oxygène pouvant conduire à une concentration en oxygène nulle provoquant ainsi l'asphyxie du milieu affectant en premier lieu la vie piscicole (tabl. 1). On peut parfois observer des dommages immédiats liès causés par un toxique puissant véhiculé par les réseaux d'eaux pluviales qui sont également les vecteurs des pollutions accidentelles.

Ce qui caractérise cette forme de pollution c'est que les effets, pour catastrophiques qu'ils soient, cessent généralement après suppression de la source de pollution. Il n'y a pas de rémanence de la pollution.

L'impact de la pollution à effet immédiat doit être appréhendé à partir des flux apportés par un événement.

\subsubsection{Effets différés}

Les effets différés sont plus difficiles à appréhender en raison de la nature des polluants que constituent les métaux lourds, les hydrocarbures et d'autres micropolluants organiques. Les principales caractéristiques de la 
Tableau 1. Intervalle de concentrations en oxygène dissous séparant la survie de la mortalité de tous les individus en fonction de la durée d'exposition et de la température (d'après Dowing et Merkens 1957)

\begin{tabular}{|l|l|l|l|r|}
\hline & \multicolumn{1}{|c|}{ Durée } & \multicolumn{2}{|c|}{ Température ${ }^{\circ} \mathrm{C}$} \\
\hline Espèce & d'exposition & $10^{\circ}$ & $16^{\circ}$ & $20^{\circ}$ \\
\hline Truite & 3,5 heures & $1,7(1,2)$ & $1,9(1,5)$ & $2,1(1,6)$ \\
arc en ciel & 3,5 jours & $1,9(1,3)$ & $3,0(2,4)$ & $2,6(2,3)$ \\
Perche & 3,5 heures & $0,7(0,4)$ & $1,1(0,6)$ & $1,2(0,9)$ \\
& 3,5 jours & $1,0(0,4)$ & $1,3(0,9)$ & $1,2(1,0)$ \\
Gardon & 3,5 heures & $0,4(0,2)$ & $0,6(0,3)$ & $1,1(0,5)$ \\
& 3,5 jours & $0,7(0,2)$ & $0,7(0,7)$ & $1,4(1,0)$ \\
\hline
\end{tabular}

Les concentrations $\mathrm{d}^{\prime} \mathrm{O}_{2}$ sont exprimées en $\mathrm{mg} / \mathrm{l}$. La première valeur est celle permettant la survie, celle entre parenthèses cause une mortalité de $100 \%$.

pollution à effets différés sont la persistance et l'accumulation d'un grand nombre de ces polluants au sein de la chaîne alimentaire. En effet, si l'on connaît certains des effets pour des expositions à de fortes concentrations dans des conditions expérimentales, il est plus difficile de rendre compte de l'influence de ces polluants dans le milieu naturel où des synergies et des antagonismes entre les différents polluants existent. Néanmoins, les apports constitués par les rejets urbains de temps de pluie ne sont pas négligeables et, bien que l'on ait des difficultés à quantifier les conséquences d'un certain nombre de ces polluants à effet différé, il paraît sage de prendre les précautions nécessaires pour diminuer ces apports de polluants.

A la différence des polluants à effets immédiats, les polluants à effets différés sont conservatifs et restent donc dans le milieu.

L'impact de la pollution à effet différé doit considérer les charges globales sur une longue période, l'année par exemple.

\subsubsection{Des objectifs de qualité}

A l'issue de ce travail d'étude, ce que l'on peut appeler des objectifs de qualité par temps de pluie doivent être définis. L'élaboration de tels objectifs présente un certain nombre de difficultés liées principalement au caractère aléatoire des événements conditionnant l'impact des rejets urbains de temps de pluie.

L'identification des causes de pollution lors des épisodes pluvieux est une étape importante car elle conditionne la démarche objectif de qualité et les moyens techniques à mettre en œuvre pour atteindre ces objectifs. Par exemple, si l'on détermine que la principale cause de pollution est l'érosion de terres agricoles, la démarche à entreprendre pour lutter contre la pollution sera très différente de celle à entreprendre lorsque la pollution est causée par des surverses de réseaux unitaires.

Les niveaux de qualité que l'on se donne comme objectif peuvent être définis à partir des usages ou à partir de critères de préservation ou de restauration d'un écosystème. Pour les cours d'eau, l'atteinte la plus évidente à la qualité d'un écosystème est constituée par les mortalités piscicoles. C'est aussi le domaine pour lequel les connaissances sont les plus avancées. Il apparaît ainsi que le parmètre le plus sensible au niveau de la pollution par les rejets urbains de temps de pluie est l'oxygène. Il semble donc logique de fixer des objectifs basés sur ce paramètre. Les Danois ont ainsi défini des niveaux de qualité par temps de pluie basés sur les durées d'exposition pour différentes espèces de poissons à des teneurs en oxygène variables en fonction de la période de retour de l'événement.

Ces niveaux de qualité par temps de pluie constituent l'un des outils nécessaire pour le dimensionnement des ouvrages de dépollution. La part de risque que l'on est prêt à admettre et les niveaux que l'on souhaite constituent l'objectif de qualité par temps de pluie.

\subsection{Les moyens techniques de lutte contre la pollution}

A partir des conclusions des différentes études et des objectifs de qualité, les actions à engager seront principalement de deux natures : des actions curatives dont l'objet est de résorber la pollution liée aux activités et à l'urbanisme existant et des actions préventives ayant pour but de ne pas aggraver la situation existante.

\subsubsection{Des actions curatives}

L'une des difficultés des actions curatives tient au fait qu'il s'agit généralement d'implanter des installations dans un milieu déjà urbanisé. Cependant les grandes options techniques peuvent être schématisées à grands traits.

Pour les réseaux unitaires, on peut considérer que l'ouvrage d'épuration principal est constitué par la station d'épuration sous réserve que celle-ci soit d'une capacité suffisante. Dans ce cas, il s'agit d'optimiser l'utilisation de 
l'ensemble réseau-station afin de tirer le meilleur parti possible de la capacité de transport du réseau et de la capacité de traitement de la station d'épuration.

Pour optimiser le fonctionnement de cet ensemble on peut mettre en place des bassins de pollution qui permettent de stocker provisoirement les eaux polluées pour les restituer ultérieurement vers la station d'épuration. On peut aussi améliorer la capacité de transport des réseaux avec des opérations d'entretien ou des modifications des déversoirs d'orage.

La dépollution au niveau des déversoirs d'orage peut être envisagée. Cependant elle présente des difficultés : la principale étant l'obtention d'une qualité de rejet suffisante pour être déversée au milieu naturel alors que le principal procédé de traitement envisagé est la décantation et que les débits de pointes peuvent être élevés.

Pour les réseaux pluviaux stricts, la principale difficulté provient de la dissémination des points de rejets qui peut entraîner une multiplication des ouvrages de traitement. La technologie aujourd'hui disponible fait également appel à la décantion et permette de prendre en compte partiellement le problème des hydrocarbures.

Pour les stations, le dimensionnement et la conception des ouvrages doivent, plus que par le passé, prendre en compte les situations de temps de pluie.

\subsubsection{La pollution liée aux urbanisations nouvelles: des actions préventives}

Dans le cas des urbanisations nouvelles, le principe de "précaution" doit être mis en œuvre : toutes les précautions visant à ne pas aggraver la situation existante, tant au niveau de la pollution que des risques d'inondation des zones aval, doivent être mises en œuvre. Pour cela la panoplie des moyens disponibles est beaucoup plus large que dans le cas des actions curatives. En effet, outre la dépollution proprement dite, une action bien plus en amont peut être entreprise au niveau des documents d'urbanisme (S.D.A.U., P.O.S., etc...). Le règlement du P.O.S. constitue l'un des outils le plus efficace pour fixer les contraintes en matière d'assainissement pluvial avec une idée directrice: la restitution, à la sortie de la parcelle aménagée, d'un débit identique à celui du terrain naturel. L'obtention du permis de construire est soumis au respect de cette contrainte par l'aménageur qui doit trouver les solutions techniques pour la respecter. Ces techniques existent : outre celle éprouvée du bassin de retenue, un certain nombre d'autres techniques dites alternatives comme les chaussées réservoirs, le stockage des eaux de toitures ou la réalisation de noues permettent de respecter cette contrainte. La réduction des débits ainsi obtenue facilite la dépollution des eaux pluviales. De plus, certaines d'entre elles, comme les bassins de retenue, ont une action de dépollution sensible. Il faut noter que l'utilisation de ces techniques de limitation des débits peuvent se traduire par des économies financières en permettant de réaliser des réseaux de dimensions réduites ainsi que des économies de terrain.

\subsubsection{La limitation de la pollution à la source : une action complémentaire}

Des actions complémentaires au niveau des sources de pollution peuvent également avoir une influence sensible sur la qualité des rejets urbains par temps de pluie. A titre d'exemple, on pensera à la pollution par le plomb qui a été réduite de manière significative aux Etats-Unis depuis la généralisation de l'usage des carburants sans plomb. Dans le même ordre d'idée, le nettoyage de voirie par aspiration constitue une voie en cours d'exploration en vue de limiter la quantité de poussière accumulée sur le sol et qui sera emportée par le ruissellement.

\subsubsection{L'entretien des ouvrages : une action indispensable}

Comme pour la dépollution des eaux usées, la condition pour obtenir de bons résultats est un entretien régulier des ouvrages de dépollution dont la fonction est d'arrêter la pollution et donc de la stocker. L'absence d'entretien conduirait rapidement à rendre ces ouvrages inefficaces et les investissements consentis seraient alors sans effet. La prise en compte des contraintes de maintenance des ouvrages dès leur conception doit être un souci permanent.

\subsubsection{Un problème en suspens: le devenir des boues}

La dépollution des rejets urbains de temps de pluie s'accompagne d'une production de boues dont le traitement et la destination n'ont pas encore trouvé de solution satisfaisante à ce jour. Ce point constitue l'un des sujets de préoccupation et de recherche de l'agence de l'eau SeineNormandie.

\section{Les moyens financiers}

Pour son $6^{\mathrm{e}}$ programme d'intervention, l'agence de l'eau Seine-Normandie consacrera à la lutte contre la pollution des collectivités un montant d'aide de 10 milliards de francs dont 1,2 milliards de francs sur cinq ans pour la dépollution des rejets urbains par temps de pluie. Soit respectivement, en montant de travaux: 17 milliards de francs pour les collectivités et 2 milliards de francs pour la dépollution des rejets urbains par temps de pluie.

Les domaines pour lesquels l'agence apporte son soutien sont les suivants :

* les études :

- études diagnostics de réseaux qui intégreront maintenant aussi bien les aspects temps sec que temps de pluie, - les schémas d'assainissement qui prendront également en compte le pluvial,

- les études milieu naturel qui permettront d'établir les objectifs de qualité par temps sec et par temps de pluie, les études préalables à la définition des projets,

- le suivi des ouvrages innovants.

Dans ce domaine les modalités d'aides sont les suivantes : en fonction des zones de 50 à $100 \%$ de prêts ou de 
subvention pour les études générales alors que pour les études d'avant projet détaillé, les conditions sont similaires à celles des travaux.

* les travaux :

- dans ce domaine, la priorité porte principalement sur la maîtrise des surverses de réseaux unitaires qui constituent la principale part de la pollution causée par les rejets urbains par temps de pluie. L'agence prendra en compte tous les ouvrages dont la fonction est de permettre une réduction de la pollution. Le montant retenu pour le finan- cement de ces ouvrages sera comparé à un prix de référence dont les modalités ne sont pas encore arrêtées.

Pour les travaux, les modalités d'aides varient en fonction de la zone considérée : le taux de subvention est de 30 , 35 ou $40 \%$. A cette subvention vient s'ajouter un prêt sur 10 ans portant sur $20 \%$ du montant retenu des travaux avec un taux d'intérêt moitié de celui pratiqué par la Caisse des Dépôts et Consignation.

Des primes pour épuration doivent progressivement voir le jour afin d'encourager l'entretien des ouvrages mis en place.

\section{Conclusion}

La prise en compte par les agences de l'eau de la pollution par les rejets urbains par temps de pluie permet d'avoir une approche globale de l'assainissement et de l'épuration pour une meilleure efficacité sur le milieu naturel. Cependant, dans ce domaine nouveau qu'est la dépollution des rejets urbains par temps de pluie, l'état actuel des connaissances doit être enrichi par l'expérience des uns et des autres. Un effort d'études, de recherche fondée notamment sur le suivi des ouvrages, et de communication doit être entrepris. Néanmoins les connaissances actuelles sont déjà suffisantes pour engager des actions de lutte contre la pollution par les rejets urbains par temps de pluie. 